\section{Relação entre força de preensão manúal e forca dé membro inferior em mulheres de meia idade: um estudo transversal}

\section{Relation between grip strength and lower limb strength in middle-aged women: a cross sectional study}

Mariana Carmem Apolinário Vieira

Carine Fernandes de Souza ${ }^{1}$

Saionara Maria Aires da Câmara ${ }^{1}$

Gabrielle Silveira Rocha Matos²

Mayle Andrade Moreira'

Alvaro Campos Cavalcanti Maciel ${ }^{1}$

\section{RESUMO}

Embora a força de preensão manual (FPM) seja utilizada para avaliação de força total do corpo, ainda há contradição na literatura acerca da capacidade preditiva da força de membro inferior (FMI) a partir da FPM. Sabe-se que a FMI é determinante na estabilidade corporal e na locomoção, sendo de extrema importância sua avaliação de forma precisa. Portanto, o objetivo desse estudo foi avaliar a correlação entre a FPM e FMI (flexores e extensores de joelho) em mulheres de meia idade. Trata-se de um estudo observacional analítico, transversal, com 395 mulheres (40 a 65 anos), avaliadas quanto à FPM e FMI (flexores e extensores de joelho). Foi utilizada correlação de Pearson, regressão linear e análise de Bland-Altman para análise dos dados, considerando significância de 5\%. A média de idade das mulheres foi de 49,75 ( $\pm 5,8)$ anos. A média da FPM e FMI (flexão e extensão de joelho) foram, respectivamente: $25,8( \pm 5,4), 14,7( \pm 4,9)$ e 16,5 $( \pm 4,5)$ Kgf. Após análise de regressão linear e análise de Bland-Altman, o valor de $\mathrm{R}^{2}$ ajustado mostrou baixa capacidade de predição da FMI a partir da FPM, com a força de extensão do joelho $\left(\mathrm{R}^{2}=0,21\right)$ apresentando valores mais elevados que a força de flexão $\left(\mathrm{R}^{2}=0,13\right)$. A FPM correlaciona-se de maneira fraca com a força de extensão e flexão de joelhos em mulheres de meia idade, evidenciando a necessidade de avaliação de diferentes segmentos corporais a fim de uma estimativa mais criteriosa nesta população.

\section{PALAVRAS-CHAVE}

Força muscular; Mulheres; Membros inferiores; Envelhecimento.

\begin{abstract}
Although the grip strength has been used to evaluate the total body's strength, there is still a contradiction in the literature about the predictive capacity of lower limb strength from the grip strenght. It is known that the lower limb strength is determinant in the body stability and locomotion, being extremely important assess it accurately. Therefore, the objective of this study is to evaluate the correlation between grip strength and lower limb strength (knee flexors and extensors) in middle-aged women. It was a cross-sectional study with 395 women (40 to 65 years old) assessed regarding the grip strength and lower limb strength (knee's flexors and extensors). It was used Pearson's correlation, linear regression and Bland-Altman analysis for data analysis and it has been taking into consideration a $5 \%$ of significance. The average age of women was $49.75( \pm 5.8)$ years. The mean of grip strength and knee's flexor and extensor strength were, respectively: $25.8 \mathrm{kgf}( \pm 5.4), 14.7 \mathrm{~kg} f( \pm 4.9)$ and 16.5 $\mathrm{kg} f( \pm 4.5)$. After linear regression and Bland-Altman analysis, the value of adjusted $R^{2}$ showed a low predictive capacity of the lower limb strength from the grip strength; and the knee extension strength $\left(R^{2}=0.21\right)$ showed values higher than the flexor strength $\left(R^{2}=0.13\right)$. The grip strength presented a weak correlation with the knee's extension and flexion strength in middle-aged women, evidencing the necessity of evaluate different body segments in order to a more careful estimate in this population.
\end{abstract}

\section{KEYWORDS}

Muscle Strength; Women; Lower Limbs; Ageing.
Rev Bras Ativ Fís Saúde p. 467-475 DOI

http://dx.doi.org/10.12820/rbafs.v.20n5p467

1 Universidade Federal do Rio Grande do Norte, Departamento de Fisioterapia, Natal, RN, Brasil.

2 Universidade Federal do Amazonas,

Departamento de Fisioterapia, Manaus, AM., Brasil. 


\section{INTRODUÇÃO}

O processo de envelhecimento é responsável por uma série de modificações fisiológicas sobre o sistema músculo-esquelético, podendo gerar déficits de força, equilíbrio e alterações na marcha ${ }^{1}$, constituindo, portanto, uma fonte de preocupação para a população ao longo desse processo ${ }^{2,3}$. Com o avanço da idade, ocorre um declínio na massa magra, responsável por uma perda significativa de força que ocasiona limitações na independência funcional e, consequentemente, na realização das atividades de vida diária (AVDs) ${ }^{1}$. Tais limitações acarretam, também, a redução de sua qualidade de vida ${ }^{4}$, visto que a capacidade de realizar as atividades comuns de vida diária sem necessidade de ajuda de outra pessoa mostra-se essencial para a manutenção da qualidade de vida ${ }^{5}$.

Esta redução, particularmente dos membros inferiores, tem sido associada ao maior risco de quedas, à diminuição da densidade mineral óssea e à maior probabilidade de fraturas, bem como, a outras alterações fisiológicas adversas, tais como, alterações no metabolismo energético e na capacidade aeróbia ${ }^{6}$. Tais mudanças fazem com que atividades como deambular, manter o equilíbrio e levantar-se de uma cadeira ou da cama estejam entre as primeiras a serem prejudicadas com a senilidade ${ }^{7}$, e progressivamente determinam a incapacidade.

Medidas de desempenho físico, incluindo medidas de mobilidade, equilíbrio e força, tem sido investigadas em relação ao seu valor preditivo para futura incidência de dependência em atividades da vida diária e incapacidade ${ }^{5,8}$. Com isso, identificar indivíduos com tais déficits pode auxiliar na detecção de um declínio funcional de forma precoce, o que possibilita um processo de intervenção de maneira otimizada 5 .

Especificamente em relação à medida de força, sua avaliação global em um indivíduo é possível ser predita a partir da FPM, por ser um método de baixo custo, simples, não invasivo e que apresenta muitas aplicações clínicas diferentes ${ }^{7,9}$. A FPM vem sendo utilizada não apenas para identificar fraqueza de membros superiores, mas também tem sido considerada representativa da força de outros grupos musculares ${ }^{10}$. Além disso, tem sido utilizada para identificar pacientes com risco aumentado de deterioração da saúde ${ }^{11}$.

Embora as alterações de força e massa muscular aconteçam em sujeitos de ambos os sexos, nota-se que estas não ocorrem na mesma taxa e idade para ambos $^{12}$. Estudo relata uma rápida perda de força nas mulheres em torno dos 50 anos, uma tendência que não é observada em homens até pelo menos os 60 $\operatorname{anos}^{12}$, possivelmente decorrente das alterações hormonais que acompanham a menopausa (marco do envelhecimento reprodutivo feminino).

Neste sentido, apesar da possibilidade de predição ser definida em vários grupos populacionais, ainda persistem lacunas de conhecimentos que devem ser mais bem investigadas, como por exemplo, em mulheres durante o processo de transição da idade adulta para a terceira idade.

Diante do exposto, embora a FPM seja utilizada para avaliação de força total do corpo, ainda há contradição na literatura acerca da capacidade preditiva da FMI a partir da $\mathrm{FPM}^{13,14}$. Sabe-se que a FMI é determinante na estabilidade corporal e na locomoção ${ }^{15}$, sendo indispensável durante o processo de envelhecimento para evitar quedas e alterações na marcha, as quais levam à incapacidade e institucionalização ${ }^{16}$, sendo de extrema importância sua avaliação de forma precisa. Portanto, o objetivo desse estudo foi avaliar a 
correlação entre a FPM e FMI (flexores e extensores de joelho) em um grupo de mulheres de meia idade.

\section{MÉTODOS}

\section{Delineamento do estudo e local de pesquisa}

Trata-se de um estudo observacional analítico de caráter transversal, realizado no município de Parnamirim/RN, região metropolitana de Natal, capital do estado do Rio Grande do Norte. Segundo o IBGE, esta cidade contém 202.456 habitantes, uma área de $123,471 \mathrm{~km}^{2}$ e é $100 \%$ urbanizada. Os dados do presente estudo foram coletados durante o período de Abril a Novembro de 2013.

\section{População e amostra}

A amostra foi composta por 395 mulheres com idade entre 40 e 65 anos, as quais foram selecionadas por conveniência após a divulgação do estudo em unidades básicas de saúde do município, em diferentes dias da semanas nos turnos matutino e vespertino.

\section{Critérios de inclusão e exclusão}

Os critérios de inclusão para o estudo foram: não apresentar doenças neurológicas e degenerativas como Parkinson, acidente vascular encefálico (AVE), doenças degenerativas medulares, fratura nos membros, processos dolorosos, ou qualquer outra condição que pudesse comprometer a mensuração dos dados identificada pelos pesquisadores no primeiro contato ou autorrelatada pela participante. A desistência ou impossibilidade de qualquer natureza em realizar qualquer uma das etapas do protocolo de pesquisa foram consideradas como critérios de exclusão.

\section{Variáveis}

As variáveis do estudo foram: idade (anos), Índice de Massa Corpórea - IMC $\left(\mathrm{kg} / \mathrm{m}^{2}\right)$, FPM (kgf) e força de extensores (kgf) e flexores de joelho (kgf).

\section{Instrumentos e procedimentos}

Os dados das mulheres que demonstraram interesse em participar foram coletados por entrevistadores treinados, em dia e horário combinado conforme agendamento prévio, utilizando questionário estruturado padronizado, de acordo com os procedimentos que serão descritos a seguir.

Foram coletados os dados sobre idade e IMC, em $\mathrm{Kg} / \mathrm{m}^{2}$ utilizando a balança Felizona ${ }^{\circledR}$, série 3.134, com divisões de $100 \mathrm{~g}$ e fita métrica Fiber glass ${ }^{\circledR}$ com divisões de $1 \mathrm{~mm}$. A FPM foi avaliada no membro dominante com dinamômetro manual hidraúlico Jamar ${ }^{\circledR}$, modelo J00105, com a voluntária posicionada em sedestação, com o ombro aduzido e em rotação neutra, cotovelo posicionado em $90^{\circ}$ de flexão, com o antebraço e punho em posições neutras ${ }^{10}$. Foram solicitadas três repetições de contrações sustentadas de cinco segundos, com intervalo de um minuto entre as repetições e utilizada a média aritmética das três medidas consecutivas ${ }^{17}$.

Para a avaliação da FMI foi utilizado o dinamômetro portátil Hoggan ${ }^{\circledR}$, modelo microFET $2^{\circledR}$. Para aferição da força de extensores de joelho, do membro inferior dominante, a voluntária foi posicionada em decúbito ventral, com 
a coxa fixada à maca por meio de faixa anelástica e joelho fletido em $90^{\circ}$ graus. O dinamômetro foi posicionado na face anterior da perna, na linha imediatamente proximal aos maléolos, fixado pelo examinador. A avaliação de flexores de joelho foi realizada na mesma posição descrita anteriormente, porém com o joelho estendido. $\mathrm{O}$ dinamômetro foi posicionado na face posterior da perna, na linha imediatamente proximal aos maléolos, fixado pelo examinador. Para ambas as medidas foram solicitadas três contrações isométricas máximas, com duração de cinco segundos, com um minuto de intervalo entre elas e considerada a média aritmética das três medidas ${ }^{17,18}$.

\section{Aspectos éticos}

O projeto foi encaminhado para avaliação pelo Comitê de Ética e Pesquisa da UFRN e foi aprovado com o parecer $\mathrm{n}^{\circ} 387.737$, sendo parte de um estudo longitudinal denominado "Influência do status menopausal e níveis hormonais na funcionalidade, desempenho muscular e composição corpórea: um estudo longitudinal". Ao comparecerem para avaliação, as mulheres foram esclarecidas quanto aos objetivos e procedimentos do estudo e solicitadas a assinar um termo de consentimento livre e esclarecido, seguindo as recomendações da Declaração de Helsinki na resolução n 466/12 do Conselho Nacional de Saúde.

\section{Análise dos dados}

Os dados foram analisados com o software SPSS versão 20.0. Foi realizada a estatística descritiva por meio das medidas de tendência central (média aritmética) e de dispersão (desvio padrão) para as variáveis quantitativas de idade, IMC e dinamometria.

A normalidade dos dados foi comprovada pela utilização do teste de Kolmogorov-Smirinov e, em seguida, foi aplicado o teste de correlação de Pearson para avaliar a correlação entre a FPM e as variáveis idade, IMC e FMI (flexores e extensores do joelho). Foram também obtidas equações de regressão linear a fim de se estimar o grau de predição das forças de flexão e extensão do joelho em função da FPM. Por fim, a concordância entre as forças de flexão e extensão de joelho e a FPM foi também analisada por meio da disposição gráfica de Bland-Altman. Em toda análise estatística foi considerado um intervalo de confiança (IC) de $95 \%$ e um $\mathrm{p}<0,05$.

\section{RESULTADOS}

No presente estudo foram avaliadas 395 mulheres, apresentando média de idade de 49,7 anos, sem ocorrência de perda amostral. Na tabela 1 estão demonstradas as demais características da amostra.

Em relação à correlação entre a FPM (kgf) e as demais variáveis: idade (r:0,174), IMC (r:0,113), força de extensão (r:0,406) e flexão de joelho (r:0,356), houve correlação significativa em todas as situações (Tabela 2). Entretanto, observou-se uma correlação fraca entre as variáveis analisadas.

As figuras 1-A e 1-C mostram os resultados de dispersão entre a FPM e a força de flexão e extensão de joelho, respectivamente. Em ambos os casos, o valor de $\mathrm{R}^{2}$ ajustado mostrou baixa capacidade de predição da FMI a partir da FPM com a força de extensão do joelho $\left(\mathrm{R}^{2}=0,21\right)$, apresentando valores mais elevados que a flexão $\left(\mathrm{R}^{2}=0,13\right)$. 
TABELA 1 - Caracterização da amostra - Natal, RN, 2014.

\begin{tabular}{lc}
\hline VARIÁVEIS & Média (DP) \\
\hline Idade (anos) & $49,7(5,8)$ \\
\hline IMC (kg/m²) & $28,7(4,9)$ \\
\hline Força de preensão (kgf) & $25,8(5,4)$ \\
\hline Força de flexão de joelho (kgf) & $14,7(4,9)$ \\
\hline Força de extensão de joelho (kgf) & $16,5(4,5)$ \\
\hline
\end{tabular}

TABELA 2 - Correlação entre a força de preensão e idade, índice de massa corporal, força de flexão e extensão de joelho - Natal, RN, 2014.

\begin{tabular}{lcc}
\hline & \multicolumn{2}{c}{ Força de preensão } \\
\cline { 2 - 3 } Variáveis & $\mathrm{R}$ & $\mathrm{P}$ \\
\hline Idade & $-0,174$ & 0,001 \\
\hline IMC & 0,113 & 0,026 \\
\hline Força de flexão de joelho & 0,356 & $<0,001$ \\
\hline Força de extensão de joelho & 0,406 & $<0,001$ \\
\hline
\end{tabular}

$\mathrm{Na}$ análise de Bland-Altman para a flexão do joelho (figura 1-B), encontrou-se uma diferença média de $-10,4( \pm 5,4) \mathrm{kgf}$, com limites de concordância de $95 \%$ variando de $-20,59$ a $-0,23 \mathrm{kgf}$. $\mathrm{Na}$ análise para extensão do joelho (figura 1-D) encontrou-se uma diferença média de -8,8 $( \pm 4,8) \mathrm{kgf}$, com limites de concordância de $95 \%$ variando de $-18,4$ a $0,58 \mathrm{kgf}$.
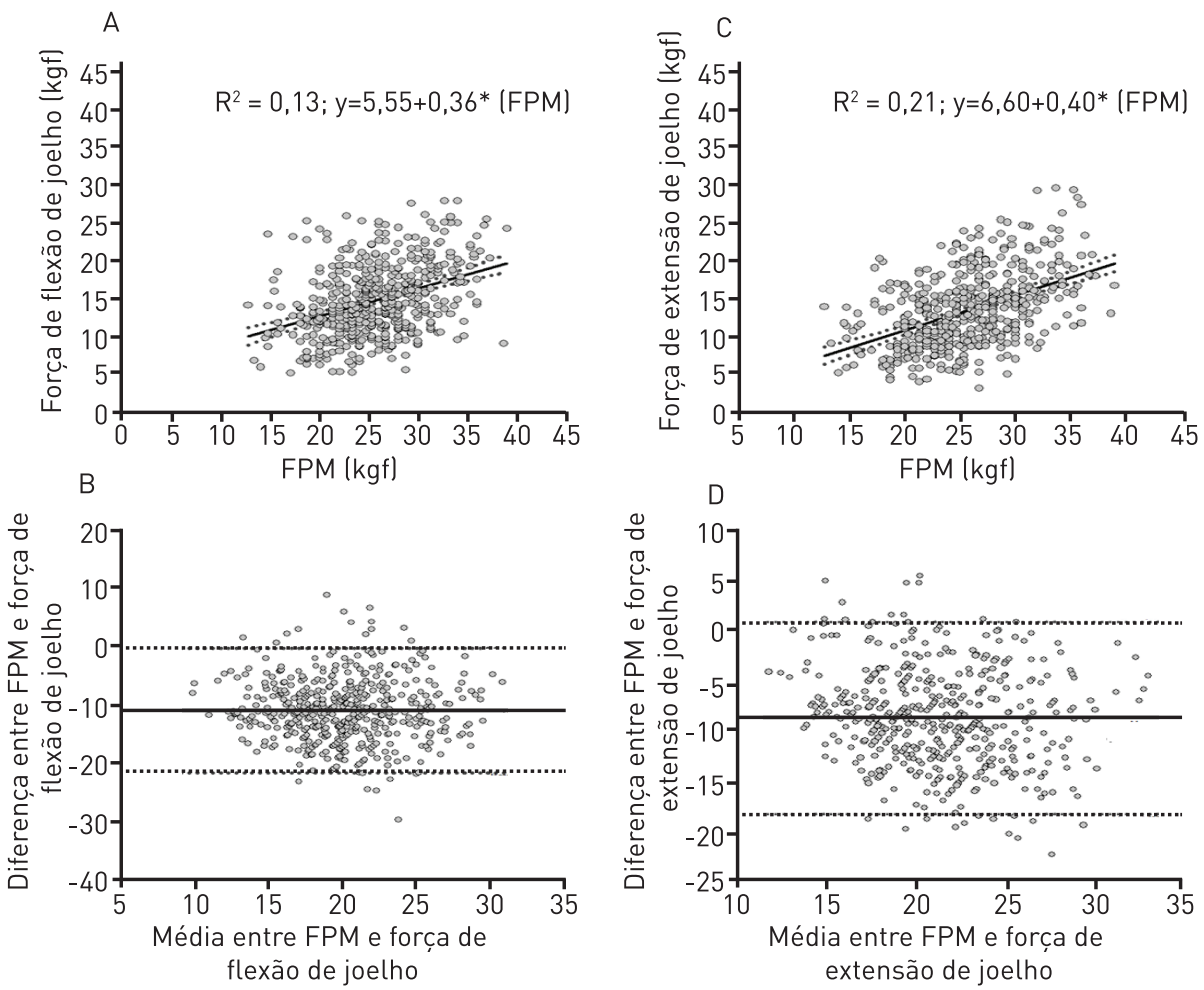

FIGURA 1 - A) Gráfico de correlação entre a FPM (força de preensão manual) e a força de flexão de joelho; B) Gráfico de Bland-Altman para FPM e força de flexão de joelho; C) Gráfico de correlação entre a FPM e a força de extensão de joelho; D) Gráfico de Bland-Altman para FPM e força de extensão de joelho.

\section{DISCUSSÃO}

A partir dos resultados do presente estudo, observou-se correlação fraca entre 
FPM e de flexores e extensores de joelho, sendo a FPM insuficiente para predizer a FMI em mulheres de meia idade.

A análise da força e da função muscular é de suma importância no processo de avaliação da aptidão física e no processo de reabilitação ${ }^{19}$. Além disso, a força é fundamental durante o processo de envelhecimento para a autonomia do idoso, devido à grande quantidade de atividades cotidianas, nas quais se necessita de níveis adequados de força para executá-las ${ }^{20}$.

A média de FPM (25,8 kgf) do presente estudo ficou abaixo de outros estudos, os quais encontraram, em geral, valores de referência que se situavam entre 26,0 e 33,0 $\mathrm{kgf}^{21,22}$. Essa diferença pode ser creditada aos diferentes perfis antropométricos entre as mulheres dos países onde as pesquisas foram realizadas (principalmente Europa e EUA), ainda, por se tratarem de mulheres brasileiras, em que maior IMC pode estar relacionado a maior força ${ }^{23} \mathrm{~A}$ amostra deste estudo foi composta por mulheres, em período próximo ao correspondente à menopausa, onde alterações hormonais unem-se a alterações de mudança de comportamento e hábitos de vida, como a inatividade física que está intimamente relacionada aos sintomas do climatério ${ }^{24}$. Em relação à força de flexão e extensão de joelho, obteve-se a média de 14,7 kgf e 16,5 kgf, respectivamente. Estudo com idosas com média de idade de 73 anos, utilizando a mesma metodologia do presente estudo, apresentaram valores de 7,3 kgf para flexão e $9,8 \mathrm{kgf}$ para extensão ${ }^{25}$. Valores estes encontrados mais baixos que o presente estudo devido à diferença de idade, entretanto, aproximadamente a mesma proporção de diferença entre a média da força de flexão e extensão do joelho.

No presente estudo, observou-se um maior valor médio de FPM em comparação com a FMI, corroborando estudos que demonstram que força muscular sofre declínio desigual ao longo do processo de envelhecimento ${ }^{2,19,26}$. Geraldes et al. (2008) ${ }^{26}$ afirmam que os membros superiores tem seu uso continuado durante todas as etapas da vida, enquanto os membros inferiores tem seu uso diminuído com a inatividade física e o envelhecimento, podendo explicar a discrepância de força entre eles.

Em um estudo correlacionando a FPM com a de flexores de joelhos entre jovens e idosos, Samuel et al. (2012) ${ }^{13}$ encontraram que a força do quadríceps possui um declínio maior com o envelhecimento em relação à $\mathrm{FPM}^{13}$. Corroborando com o estudo longitudinal de Matsudo et al. (2004) ${ }^{2}$, com mulheres de 50 a 82 anos, no qual foi realizado o teste de impulsão vertical sem o uso das mãos para avaliar a FMI, foi encontrado que a mesma diminuiu em torno de $13 \%$ e a de membros superiores se manteve estável, após 4 anos $^{2}$. Foi observada uma fraca correlação entre a FPM e a FMI $(\mathrm{r}: 0,42)^{2}$, similarmente aos resultados encontrados.

No presente estudo, tanto o valor de $\mathrm{R}^{2}$ ajustado quanto a análise de Bland-Altman mostram uma baixa capacidade de predição das forças de membros inferiores a partir da FPM. No estudo de Benedetti et al $(2010)^{27}$, no qual analisava diferentes testes de força em idosos praticantes de atividade física, foi identificada a correlação entre a FPM e a FMI, todavia, todos apresentaram baixa correlação ${ }^{27}$. Tais achados ressaltam a necessidade de avaliação de diferentes segmentos corporais a fim de uma estimativa mais criteriosa a respeito da força durante a senilidade.

Corroborando o presente estudo, Felicio et al. (2014) ${ }^{14}$, afirmam não haver correlação entre as variáveis avalidas de FPM e força dos flexores e extensores 
de joelho. Bohannon et al. $(2012)^{21}$ consideram a medida de FPM insuficiente para caracterizar a força global, já que não representa, muitas vezes, a dificuldade em realizar atividades que utilizam membros inferiores, como sentar e levantar ${ }^{21}$. Esses resultados sugerem que embora seja amplamente utilizada, a FPM não é uma medida adequada para predizer a FMI, devendo ser usada com cautela ${ }^{14}$.

Em concordância, Bassey $(1998)^{28}$ considera que a FPM não é suficiente para medir a força total, devido às desvantagens como: a) os músculos avaliados não são essenciais para tarefas que envolvem o suporte do peso corporal; b) as associações entre a FPM e a força de diferentes grupamentos musculares são, geralmente, fracas ou, no máximo, moderadas; c) contrações estáticas raramente são necessárias às atividades do cotidiano ${ }^{28}$. Portanto, os resultados encontrados no presente estudo de que a FPM apresenta correlação fraca com a FMI corroboram o estudo de Bassey (1998), Bohannon (2012) e Felicio (2014).

As limitações do estudo incluem o seu caráter transversal, uma vez que um estudo longitudinal poderia verificar de forma mais eficaz se a perda de força ao longo dos anos é realmente diferente entre os membros superiores e inferiores. A amostra foi composta por conveniência e o posicionamento para avaliação da força dos flexores e extensores de joelho não pôde ser realizado em posição funcional. A variabilidade metodológica quanto ao posicionamento e instrumentos utilizados para avaliação da FMI torna limitada a discussão em relação à comparação dos valores médios dessa variável. Participantes são mulheres de meia-idade do Nordeste do Brasil, portanto, deve-se ter cautela para inferir tais resultados para outras populações. Por fim, ainda como limitação, o presente estudo não avaliou variáveis relacionadas à atividade física, impedindo a realização de análises nesse aspecto. Sugere-se a realização de novos estudos que considerem a atividade física como covariável para confirmação dos resultados do presente estudo.

Entretanto, embora existam limitações, ressalta-se a importância do presente estudo, que a partir de análises metodológicas válidas, comparou variáveis de força com unidades semelhentes. Além disso, não se tem conhecimento até o presente momento, de estudo com a presente abordagem em mulheres de meia idade do Nordeste brasileiro.

\section{CONCLUSÃO}

A partir dos resultados do presente estudo, observou-se uma correlação fraca entre FPM e de flexores e extensores de joelho, sendo a FPM insuficiente para predizer a FMI em mulheres nessa faixa etária. Demonstrou-se, com isso, a necessidade da avaliação de diferentes segmentos corporais com o intuito de uma estimativa mais criteriosa a respeito da força durante o processo de envelhecimento para prevenção dos desfechos adversos decorrentes desse período.

\section{Contribuição}

MCAV, CFS, SMAC, GSRM, MAM e ACCM participaram da concepção do projeto, desenho do estudo, análise e interpretação dos dados. MCAV, SMAC, MAM e ACCM participaram da redação do artigo e revisão crítica relevante do conteúdo intelectual. MCAV, MAM e ACCM participaram da aprovação final da versão a ser publicada. 


\section{REFERÊNCIAS}

1. Faria JC, Machala CC, Dias RC, Dias JMD. Importância do treinamento de força na reabilitação da função muscular, equilíbrio e mobilidade de idosos. Acta Fisiátr. 2003;10(3):133-7.

2. Matsudo SM, Marin RV, Ferreira MT, Araújo TL, Matsudo V. Estudo longitudinaltracking de 4 anos - da aptidão física de mulheres da maioridade fisicamente ativas. $\mathrm{R}$ Bras Ci e Mov. 2004;12(3):47-52.

3. Anton MM, Spirduso WW, Tanaka H. Age-related declines in anaerobic muscular performance: weightlifting and powerlifting. Med Sci Sports Exerc. 2004;36(1):143-7.

4. Lobo AJS, Santos L, Gomes S. Nível de dependência e qualidade de vida da população idosa. Rev Bras Enferm. 2014;67(6):913-9.

5. Wennie-Huang WN, Perera S, VanSwearingen J, Studenski S. Perfomance measures predict onset of activity of daily living in community-dwelling older adults. J Am Geriatr Soc. 2010;58(5):844-52.

6. Carvalho J, Oliveira J, Magalhães J, Ascensão A, Mota J, Soares JMC. Força muscular em idosos II - Efeito de um programa complementar de treino na força muscular de idosos de ambos os sexos. Rev Port Cien Desp. 2004;4(1):58-65.

7. Oliveira JP, Neri AL. Velocidade da marcha, força de preensão e saúde percebida em idosos: dados da rede FIBRA Campinas, São Paulo, Brasil. Ciênc Saúde Colet. 2014;19(8):3343-53.

8. Santos RG, Tribess S, Meneguci J, Bastos LLAG, Damião R, Júnior JSV. Força de membros inferiores como indicador de incapacidade funcional em idosos. Motriz: rev. educ. fis. 2013;19(3):35-42.

9. Sallinen J, Stenholm S, Rantanen T, Heliövaara M, Sainio P, Koskinen S. Hand-Grip Strength Cut-Points to Screen Older Persons at Risk for Mobility Limitation. J Am Geriatr Soc. 2010;58(9):1721-6.

10. Moreira D, Álvarez RRA, Gogoy JR, Cambraia AN. Abordagem sobre preensão palmar utilizando o dinamômetro JAMAR®: uma revisão de literatura. R Bras Ci e Mov. 2003;11(2):95-9.

11. Abizanda P, Navarro JL, Garcia-Tomas MI, Lopez-Jimenez E, Martinez-Sanchez E, Paterna G. Validity and usefulness of hand-held dynamometry for measuring muscle strength in community-dwelling older persons. Arch Gerontol Geriatr. 2012;54(1):21-7.

12. Maltais ML, Desroches J, Dionne IJ. Changes in muscle mass and strength after menopause. J Musculoskelet Neuronal Interact. 2009;9(4):186-97.

13. Samuel D, Wilson K, Martin HJ, Allen R, Sayer AA, Stokes M. Age-assotiated changes in hand grip and quadriceps muscle strength ratios in healthy adults. Aging Clin Exp Res. 2012;24(3):245-50.

14. Felicio DC, Pereira DS, Assumpção AM, Jesus-Moraleida FR, Queiroz BZ, Silva JP, et al. Poor correlation between handgrip strength and isokinetic performance of knee flexor and extensor muscles in community-dwelling elderly women.Geriatr Gerontol Int. 2014;14:185-9.

15. Fiatarone-Singh MA. Body composition and weight control in older adults. In: Lamb DR, Murray R (eds). Perspectives in exercise science and sports medicine: exercise, nutrition and weight control. Carmel: Cooper; 1998. 243-88.

16. Kron M, Loy S, Sturm E, Nikolaus T, Becker C. Risk indicators for fall in institutionalized frail elderly. Am J Epidemiol 2003;158(7):645-653.

17. Pereira LSM, Narciso FMS, Oliveira DMG, Coelho FM, Souza DG, Dias RC. Correlation between manual muscle strength and interleukin-6 (IL-6) plasma levels in elderly community-dwelling women. Arch Gerontol Geriatr. 2009;48(3):313-6.

18. Martin HJ, Yule V, Syddall HE, Dennison EM, Cooper C, Sayer AA. Is hand-held dynamometry useful for the measurement of quadriceps strength in older people? A comparison with the gold standard biodex dynamometry. Gerontology. 2006;52(3):154-9.

19. Monteiro WD, Amorim PRS, Farjalla R, Farinatti PTV. Força muscular e características morfológicas de mulheres idosas praticantes de um programa de atividades físicas. Rev Bras Ativ Fís Saúde. 1999;4(1):20-8.

20. Borges LJ, Cardoso AS, Benedetti TRB, Mazo GZ, Lopes MA, Borgatto AF. Teste de resistência de força de membros superiores para idosos: comparação entre halteres com pesos diferentes. Rev Bras de Cineantropom Desempenho Hum. 2008;10(3):261-5. 
21. Bohannon RW, Peolsson A, Massy-Westropp N, Desrosiers J, Bear-Lehman J. Reference values for adult grip strength measured with a Jamar dynamometer: a descriptive meta-analysis. Physiotherapy. 2006;92(1):11-5.

22. Cote MP, Kenny A, Dussetschleger J, Farr D, Chaurasia A, Cherniack M. Reference Values for Physical Performance Measures in the Aging Working Population. Hum factors. 2014;56(1):228-42.

23. 23- Rolland Y, Lauwers-Cances V, Pahor M, Fillaux J, Grandjean H, Vellas B. Muscle strength in obese elderly women: effect of recreational physical activity in a crosssectional study. Am J Clin Nutr. 2004;79(4):552-7.

24. Guimarães ACA, Baptista F. Relationship between physical activity and menopausal symptoms. Rev Bras Ativ Fís Saúde. 2011;16(2):144-9.

25. Ching-Yi W, Olson SL, Protas EJ. Test-retest strength reliability: Hand-held dynamometry in community-dwelling elderly fallers. Arch Phys Med Rehabil. 2002;83(6):811-5.

26. Geraldes AAR, Oliveira ARM, Albuquerque RB, Carvalho JM, Farinatti PTV. A força de preensão manual é boa preditora do desempenho funcional de idosos frágeis: um estudo correlacional múltiplo. Rev Bras Med Esporte. 2008;14(1):12-6.

27. Benedetti TRB, Meurer ST, Borges LJ, Conceição R, Lopes MA, Morini S. Associação entre os diferentes testes de força em idosos praticantes de exercícios. Fit Perf J. 2010;9(1):52-7.

28. Bassey EJ. Longitudinal changes in selected physical capabilities: muscle strength, flexibility and body size. Age Ageing. 1998;27(3):12-6.

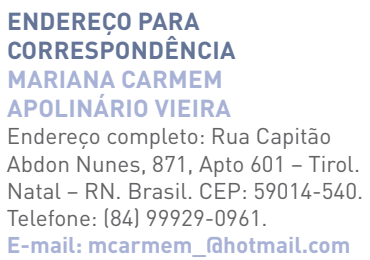

